\title{
Analysis of Human-Land Coupled Bearing Capacity of Qiangtang Meadow in Northern Tibet Based on Fuzzy Clustering Algorithm
}

\author{
ShiBao Li $\mathbb{D}$ \\ School of Science, Tibet University, Lhasa 850000, China \\ Correspondence should be addressed to ShiBao Li; 15246205666@utibet.edu.cn
}

Received 16 April 2019; Accepted 11 August 2019; Published 7 May 2020

Academic Editor: Georgios I. Giannopoulos

Copyright ( $\odot 2020$ ShiBao Li. This is an open access article distributed under the Creative Commons Attribution License, which permits unrestricted use, distribution, and reproduction in any medium, provided the original work is properly cited.

\begin{abstract}
Aiming to address the problems of high energy consumption, low efficiency, low correlation between the analyzed and actual results, and poor rationality of research indexes in current methods of analysis of human-land coupled bearing capacity of meadows, a novel method of human-land coupled bearing capacity analysis of Qiangtang meadow in northern Tibet, based on fuzzy clustering algorithm, is proposed. Basic geographic information data in Tibet were acquired, the collected data images were registered by ENVI4.2 software, and the collected data were vectorized by ArcGIS 9.3 software to construct a basic geographic information database in Tibet. Based on the frequency domain processing algorithm, the geographic information image was suppressed by noise and filtered by using a high-pass filter to realize the geographic information data processing in the study area. The human-land coupled bearing capacity analysis of Qiangtang meadow in northern Tibet was evaluated through fuzzy clustering, bearing capacity evaluation, and bearing capacity calculation under the sharing of closure. The experimental results showed that the average running energy consumption of the method was $81 \mathrm{~J}$, and $97 \%$ of the analyzed results were consistent with the actual situation. These results indicate that the operation efficiency of the method is high, and the rationality coefficient of the research index is large. The proposed method has superior performance and feasibility.
\end{abstract}

\section{Introduction}

As a very unique geographical unit, Tibet has the highest altitude and largest area in the world and is also the largest area of natural grassland in China. Influenced by high altitude environmental conditions, climatic conditions in the plateau is not divided into four seasons but show a significant difference between cold and warm seasons [1]. The nongrowing season is cold and long, while the growing season has a relatively high temperature and sufficient precipitation. The typical characteristics of hydrothermal synchronization are very conducive to synthesis and accumulation of nutrients in forage. Cordyceps sinensis excavation and grazing are the ways by which grassland in Tibet is utilized [2]. However, it was found that Cordyceps sinensis excavation and grazing were also the main human perturbing factors that led to degradation of grassland, increased spatial heterogeneity of community, and caused functional changes in plant community and decline of species diversity. With the increase of grazing intensity, the abundance of some species with poor palatability and grazing tolerance decreased, resulting in a decline in species diversity and emergence of a single plant community structure [3]. Consequently, the constant scale of grazing livestock and continuous decrease of grazing grassland area will result in the decrease of species diversity and grassland quality, which will further lead to serious degradation of grassland [4]. Besides, overgrazing may reduce the nutrient content and yield of forage. Based on the aforementioned, it is imperative to analyze the human-land coupled bearing capacity of meadows.

Hu and Huang [5] artificially measured the running state of recreational environment of the mountain meadow; selected 20 evaluation indexes from three aspects of elasticity, support, and resistance; constructed the evaluation index system of recreational environmental carrying capacity of mountain meadow; determined the threshold range of each index and each carrying capacity index based on empirical 
data and experimental data; and finally constructed the corresponding carrying capacity index model. On this basis, an evaluation index system and a model method were used to evaluate the recreational environmental carrying capacity of mountain meadow in Wugong Mountain. The results showed that the index selection of this method was reasonable, but there was a problem of high energy consumption in the operation [5]. In order to study the reinforcement mechanism of the meadow soil and stress distribution rule in the meadow soil foundation under embankment, Feng et al. [6] selected a meadow soil foundation under the embankment of Tupo Highway in the Zhaosu Reclamation Area, Yili, Xinjiang, as the research object; mixed peat soil and plain soil in different proportions; tested the density, moisture content, cohesion, and internal friction angle of the mixed soil through indoor tests; and used the fuzzy number algorithm. The similarity between mixed soil and meadow soil was theoretically evaluated, and similar soil mass of meadow soil was determined. Based on a large-scale model test in laboratory and considering the shape of embankment and the effect of flexible loading, filling process of embankment on homogeneous soft soil foundation and meadow soil foundation was simulated, and the law of stress distribution in the foundation with or without meadow soil layer under the embankment was studied. The experimental results showed that the method was more comprehensive, but the analysis efficiency was low [6]. Sun et al. [7] explored the reversal succession of alpine meadow-alpine grassland in the Zoige Huahu Wetland National Nature Reserve as a research object. Combining the current situation of grassland utilization and microtopographic distribution, five alpine degraded grassland areas were selected for investigation and correlation analysis on vegetation survey, rodent density, and soil physical and chemical properties; three kinds of hydrolytic enzyme activities and evaluation of microbial community quantity were performed. The results showed that the method was simple and easy to implement, but there was a problem of low correlation between the analyzed and actual results [7]. Zhao et al. [8] used In VEST to simulate the dynamic changes of carbon storage in Tibetan ecosystem from 2001 to 2010, and attribution analysis of carbon storage dynamics was carried out for two aspects: land cover type transfer and land cover carbon density change. It was found that Tibet's carbon storage increased by $0.5 \times 108 t$ from 2001 to 2010. Carbon storage in Northwest and Southeast Tibet changed significantly, while that in central and northern Tibet was relatively stable. Carbon storage increased in pastoral areas but decreased in agricultural, semiagricultural, and semipastoral areas. Carbon storage in water conservation areas and wind-proof and sand-fixing areas increased greatly, while biodiversity reserves and characteristic industrial areas decreased. Furthermore, carbon storage of grassland continued to grow, carbon storage of forests and sparse vegetation steadily declined, and carbon storage of shrubs declined. The experimental results showed that the energy consumption of this method was low, but the source of the selected research indexes was not very ideal, which led to the poor rationality of the research indexes [8].
Aiming at the problems existing in the current research in this field, a method for human-land coupled bearing capacity analysis of Qiangtang meadow in northern Tibet based on fuzzy clustering algorithm has been proposed. The details of the process are as follows:

(1) ENVI4.2 software was used to register the collected data and ArcGIS 9.3 was used to vectorize the collected data, which provides a basis for the analysis of human-land coupled bearing capacity of Qiangtang meadow in northern Tibet

(2) Based on the frequency domain processing algorithm, the noise of the geographic information image was suppressed, and the reconstructed geographic information image was filtered by using a high-pass filter to realize the preprocessing of the collected data

(3) Fuzzy clustering algorithm was used to analyze the human-land coupled bearing capacity of Qiangtang meadow in northern Tibet, and the final results of the human-land coupled bearing capacity of the meadow were obtained

(4) The proposed method was verified by experiments and discussions

(5) The full text is summarized, and suggestions are put forward for future development

\section{Materials and Methods}

2.1. Obtaining Basic Geographic Information Data in Tibet. According to the characteristics of the surface landscape in the study area and taking a topographic map of Tibet as a reference, the collected data images were registered using ENVI4.2 software and divided according to administrative boundaries. Projection and coordinate systems were defined for spatial overlay analysis. Secondly, with the support of ArcGIS 9.3, the basic database of geographical information in Tibet was established. The data format used was SHAP. Finally, using the mapping function of ArcG1S software, a geographical thematic map of Tibet was generated. The topographic and geomorphological maps, rainfall distribution map, and water resources distribution map are represented in Figures 1(a)-1(c), respectively.

It is well known that Qiangtang meadow in northern Tibet is a typical region of Tibet [9]. The topographic and geomorphological map and rainfall and water resources distribution in Figure 1 are part of the data collection of Qiangtang meadow, which can provide a basis for the analysis of human-land coupled carrying capacity of this region.

2.2. Information Data Processing. Because the information and data collected in Section 2.1 are presented in the form of images, it is necessary to process the data to a certain extent to meet the needs of human-land coupled bearing capacity analysis of Qiangtang meadow in northern Tibet.

Usually, the target in the collected geographic image is mainly affected by speckle noise. The gray probability density expression of this kind of noise is as follows: 


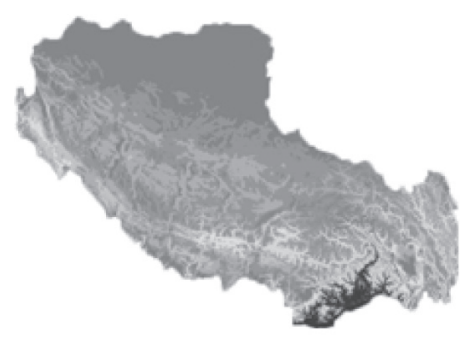

(a)

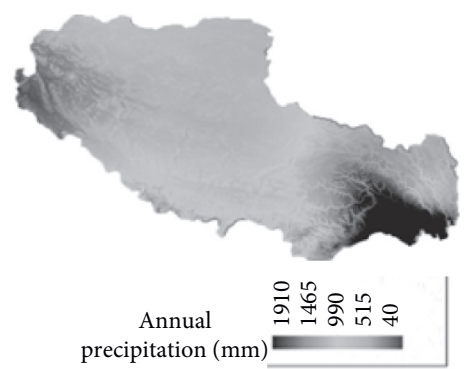

(b)

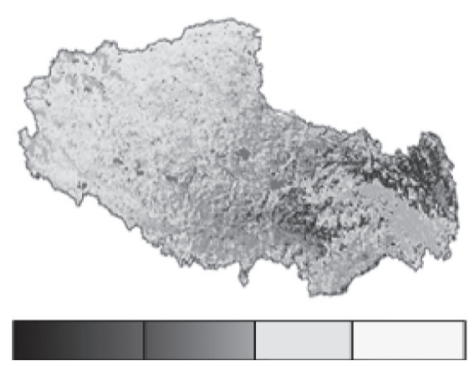

(c)

FIgURE 1: Geographical thematic maps of Tibet. (a) Topographic and geomorphological map of Tibet. (b) Rainfall distribution map of Tibet. (c) Distribution of water resources in Tibet.

$$
P(I)=\int_{I}^{\infty} p_{I}(\tau) \mathrm{d} \tau=\exp \left(-\frac{I}{\langle I\rangle}\right),
$$

where $P(I)$ represents the probability that the intensity of gray scale is greater than $I$ and is the negative exponential distribution of speckle noise after normalization of the gray scale.

The background of the geographic image is mainly affected by additive Gaussian noise. The gray probability density of this kind of noise is expressed as follows:

$$
P(I)=\int_{I}^{\infty} p_{I}(\tau) d \tau=\frac{1}{\alpha} \int_{I}^{\infty} \exp \left[-\frac{\left(\tau-I_{0}\right)^{2}}{2 \alpha^{2}}\right] d \tau,
$$

where $I_{0}$ represents the intensity of noise-free signal, and $\alpha$ represents the normalized constant. Its expression is as follows:

$$
\alpha=\int_{I}^{\infty} \exp \left[-\frac{\left(I-I_{0}\right)^{2}}{2 \alpha^{2}}\right] \mathrm{dI} .
$$

In view of the abovementioned analysis of the characteristics of geographic images, research was carried out in both time and frequency domains. By comparing the experimental results of various algorithms, such as noise filtering and target enhancement, we chose a processing algorithm based on the frequency domain to suppress the noise of geographic information images, which can effectively improve the SNR of the echo signal. High-pass filtering of the reconstructed geographic information image can improve image contrast and highlight target contour [10]. Frequency domain noise filtering: to suppress backscattering noise in the frequency domain, target echo signal was retained and the signal-to-noise ratio was improved; the main idea was to suppress the larger coefficients near the center of the logarithmic spectrum, while the smaller spectral coefficients remain unchanged.

$$
F^{\prime}\left(u, v_{i}\right)=\left\{\begin{array}{l}
\bar{F} \cdot P(I), F\left(u, v_{i}\right) \geq K_{1} \bar{F} \\
F\left(u, v_{i}\right) P(I), F\left(u, v_{i}\right)<K_{1} \bar{F}
\end{array}\right.
$$

where $\mathrm{F}\left(u, v_{i}\right)$ represents the logarithmic Fourier transform of the image, $\bar{F}$ represents the mean of the logarithmic spectrum, $v_{i}$ represents the $i$ th column in the image frequency domain, $u$ represents a row in the image frequency domain, and $K_{1}$ represents the proportional coefficient.

High-pass filter selection improves the Butterworth high-pass filter. The Butterworth high-pass filter can greatly reduce the brightness of the image at low cut-off frequency, so it needs to be improved [11]. The improved expression is

$$
H^{\prime}(u, v)=a+b H(u, v)
$$

where

$$
H(u, v)=\frac{1}{1+\left[D_{0} / D(u, v)^{2 n}\right]}, \quad D(u, v) \neq 0 .
$$

In equations (5) and (6), $H^{\prime}(u, v)$ represents the processed geographic information image, $H(u, v)$ represents the original image, and $a$ and $b$ represent two constant adjustment parameters. $a$ is an offset generated by the transfer function, and the zero frequency and low frequency information near $D(u, v)=0$ is not completely suppressed. $D 0$ represents the cut-off frequency, which is an important parameter for filtering. $n$ represents the order of the Butterworth filter. When the parameter $b$ is greater than 1 , the transfer function can be amplified, i.e., the high frequency information can be strengthened, so as to improve the efficiency of bearing capacity analysis and reduce the energy consumption of analysis. The expression of $D(u, v)$ in equation (6)is as follows:

$$
D(u, v)=\sqrt{\left(u-\frac{n}{2}\right)^{2}+\left(v-\frac{n}{2}\right)^{2}} .
$$

Equation (7) denotes the distance from the point $(u, v)$ where a Fourier coefficient is located to the positive center of the frequency rectangle.

2.3. Analysis of Human-Land Coupled Bearing Capacity of Qiangtang Meadow in Northern Tibet Based on Fuzzy Clustering Algorithm. In order to analyze the human-land coupled bearing capacity of Qiangtang meadow in northern Tibet, not only the environmental problems of the region itself but also the various parts that influence the humanland coupled bearing capacity $[12,13]$ should be considered. The evaluation index system of Qiangtang meadow carrying 
capacity should include three parts: population resource subsystem, natural resource subsystem, and environment resource subsystem. According to the available data and by comparing the horizontal and vertical data as well as the existing data using the Delphi method, the weight values at all levels of the index system were finally determined on the basis of 20 experts' opinions on the important ranking and weight assignment of indexes. Based on these results, the evaluation index system of resource carrying capacity was constructed, so as to enhance the rationality of the research indexes. Table 1 shows the index system of human-land coupled bearing capacity of Qiangtang meadow in northern Tibet.

The subsystem of population and resources in Table 1 is defined as $x 1$, the subsystem of natural resources is defined as $x 2$, and the subsystem of environment and resources is defined as $x 3$. The impact index system of human-land coupled carrying capacity of Qiantang meadow in northern Tibet can be defined as $\mathrm{X}=\{\mathrm{x} 1, \mathrm{x} 2, \mathrm{x} 3\}$.

The important value $x_{i^{\prime} j^{\prime}}^{\prime}$ of human-land coupled bearing capacity in different meadow areas in the Tibet geographic information image $H^{\prime}(u, v)$ is different, and the expression of $x_{i^{\prime} j^{\prime}}^{\prime}$ is as follows:

$$
x_{i^{\prime} j^{\prime}}^{\prime}=d_{i^{\prime} j^{\prime}}+f_{i^{\prime} j^{\prime}}+c_{i^{\prime} j^{\prime}} .
$$

In equation (8), $d_{i^{\prime} j^{\prime}}$ represents the relative carrying capacity of a region, $f_{i^{\prime} j^{\prime}}$ represents the relative frequency, and $c_{i^{\prime} j^{\prime}}$ represents the relative coverage.

The calculation results of the important values of equation (8) are treated as the original data matrix $X^{\prime}=\left[x_{i^{\prime} j^{\prime}}^{\prime}\right]$, and the similarity coefficient $r_{i^{\prime} j^{\prime}}$ between meadows in the image is calculated from the inner product-angle cosine equation of the normalized module:

$$
r_{i^{\prime} j^{\prime}}=\frac{\sum_{k^{\prime}=1}^{i^{\prime}=0} x_{k^{\prime} i^{\prime}}^{\prime} x_{k^{\prime} j^{\prime}}^{\prime}}{\sqrt{\sum_{k^{\prime}=1}^{i^{\prime}=0} x_{k^{\prime} i^{\prime}}^{\prime 2} \cdot \sum_{k^{\prime}=1}^{i^{\prime}=0} x_{k^{\prime} j^{\prime}}^{\prime 2}}}
$$

where $x_{k^{\prime} j^{\prime}}^{\prime}$ represents the corresponding value of row $k^{\prime}$ and column $j^{\prime}$ in the table of important values. According to equation (9), the fuzzy similarity function can be defined as

$$
R=\left(r_{i^{\prime} j^{\prime}}\right)_{k^{\prime} \times k^{\prime}}
$$

For effective analysis of the human-land coupled bearing capacity of Qiangtang meadow, the calibration relationship among different meadow areas needs to be equivalent, while the fuzzy similarity function $R$ only reflects the reflexivity and symmetry of the relationship and does not possess the property of transferability. Therefore, the $R$ should be modified to obtain the fuzzy equivalence function as follows:

$$
R^{2 u}=\left(r_{i^{\prime} j^{\prime}}^{2 u}\right)_{k^{\prime} \times k^{\prime}}
$$

The results of equation (11) can be used to cluster the human-land coupled carrying capacity region of the Tibetan geographic information data $H^{\prime}(u, v)$ as follows:

$$
C=\frac{R^{2 u} \cdot H^{\prime}(u, v) \cdot \mu}{\left(X+X^{\prime}\right)},
$$

where $\mu$ represents the adjusting threshold of the regional cluster of the human-land coupled bearing capacity in the Tibetan geographic information data, and the value controlled in the interval of $[0.3,0.4]$ can improve the accuracy of the analyzed data of the human-land coupled bearing capacity of Qiangtang meadow in northern Tibet.

The results of equation (12) were identified by multiobjective fuzzy pattern recognition, and the evaluated results of human-land coupled bearing capacity of the meadow in northern Tibet are represented as an output:

$$
y_{l}=\frac{1}{1+\left[\left(w_{l^{\prime}}\left|R^{2 u}-1\right|\right) / C\right]},
$$

where $y l$ represents the bearing capacity evaluation results of target $l$ and $w_{l^{\prime}}$ represents the weight.

In fact, the meadow has a certain closure effect. Even though excavation of Cordyceps sinensis and excessive artificial yak grazing have destroyed the soil and water ecological environment of the meadow, the closure effect of the meadow layer will improve the bearing capacity of the foundation to a certain extent, depending on the gravity and shear strength of the meadow layer and the impact width of the closure effect of the meadow layer $[14,15]$. Therefore, in the process of analyzing the humanland coupled bearing capacity of meadow, it is necessary to consider the effect of closure on the width $\Delta \mathrm{B}$, which should be the vertical additional stress distribution width $B$ ' at the interface between meadow layer and underlying soft soil minus the equivalent load width $B$. It is assumed that other factors causing damage to the soil and water ecological environment of the meadow due to Cordyceps sinensis excavation and excessive artificial yak grazing are $h$. The ultimate results of human-land coupled bearing capacity of Qiangtang meadow in northern Tibet are as follows:

$$
\begin{aligned}
Q & =\frac{\left[2 \int_{0.5}^{0.5 B^{\prime} / B} q(X) h\right]}{B} \cdot y_{l} \\
& =\frac{2 \cdot 0.5 \cdot\left(\gamma_{1} H^{\prime}+\vartheta \cdot \sin \theta\right) \cdot\left(0.5 B^{\prime}-0.5 B\right) h}{B} \cdot y_{l},
\end{aligned}
$$

where $Q$ represents the ultimate result of human-land coupled bearing capacity analysis of Qiangtang meadow in northern Tibet, $q(X)$ represents the stress distribution function of top surface of soft soil under ultimate bearing capacity, $\gamma 1$ represents the bulk density of the meadow layer, $H^{\prime}$ represents the thickness of the meadow layer, $\vartheta$ represents the shear strength of the meadow layer, and $\theta$ represents the angle between the shear plane and the horizontal plane.

In summary, the results of human-land coupled bearing capacity of Qiangtang meadow in northern Tibet are in good 
TABLE 1: Index system of human-land coupled bearing capacity of Qiangtang meadow in northern Tibet.

\begin{tabular}{|c|c|c|c|}
\hline Index & Index & Weight & Index \\
\hline \multirow{5}{*}{ Population resource subsystem } & \multirow{3}{*}{ Population situation } & \multirow{3}{*}{0.7} & Total population \\
\hline & & & Natural growth rate \\
\hline & & & Average life expectancy \\
\hline & \multirow{3}{*}{ Population burden } & \multirow{3}{*}{0.3} & Child dependency ratio \\
\hline & & & Old-age dependency ratio \\
\hline \multirow{7}{*}{ Natural resource subsystem } & & & Per capita arable land area \\
\hline & \multirow[t]{2}{*}{ Land resource } & \multirow[t]{2}{*}{0.35} & Crop sown area \\
\hline & & & Land use rate \\
\hline & Water resource & 0.35 & Per capita water resources \\
\hline & \multirow{2}{*}{ Energy resource } & \multirow{2}{*}{0.2} & Electricity consumption \\
\hline & & & GDP energy consumption \\
\hline & Forest resource & 0.1 & Forest coverage \\
\hline \multirow{6}{*}{ Environmental resource subsystem } & \multirow{5}{*}{ Environmental pollution control } & \multirow{5}{*}{0.7} & Production of industrial solid waste \\
\hline & & & Discharge of industrial wastewater \\
\hline & & & Industrial wastewater discharge as per standard \\
\hline & & & Industrial exhaust emissions \\
\hline & & & Investment in environmental pollution control as a proportion of GNP \\
\hline & Air environmental quality & 0.3 & - \\
\hline
\end{tabular}

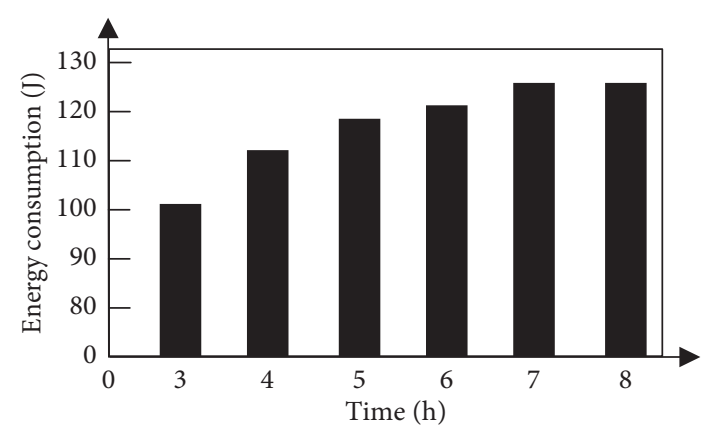

(a)

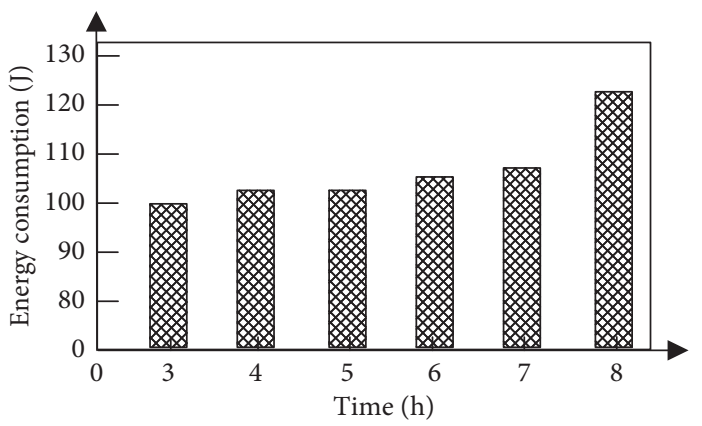

(c)

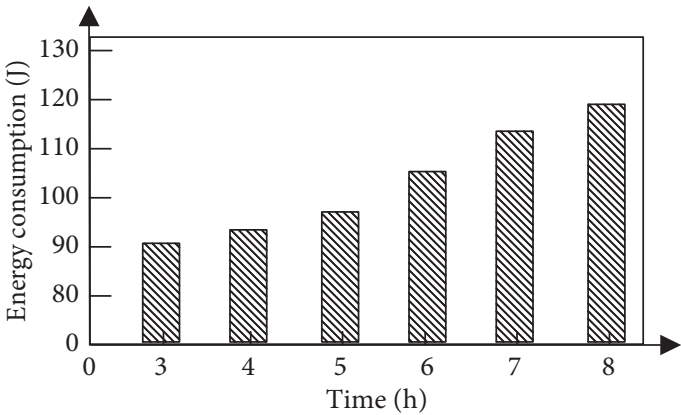

(b)

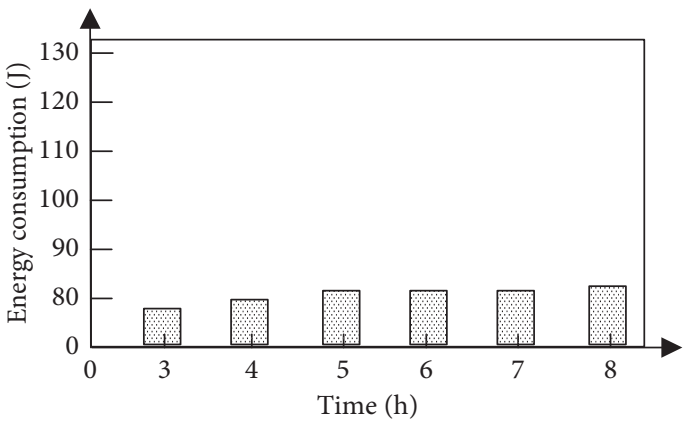

(d)

FIGURE 2: Energy consumption comparisons of different methods. (a) Energy consumption of method 1. (b) Energy consumption of method 2. (c) Energy consumption of method 3. (d) Energy consumption of human-land coupled bearing capacity analysis method for Qiangtang meadow in northern Tibet based on fuzzy clustering algorithm.

agreement with the actual situation evaluated by means of fuzzy clustering, bearing capacity evaluation, and bearing capacity calculation considering the closure effect.

\section{Results}

The Qiangtang Plateau is an integral part of the QinghaiTibet Plateau. It is also the largest inland flow area of the plateau. It is the second largest lake area in China and the highest inland lake area in the world. Meadow vegetation is extensive in this area, but the ecological environment damage of soil and water caused by Cordyceps sinensis excavation and excessive artificial yak grazing makes it imperative for experts in this field to study the human-land coupled carrying capacity. In order to verify the validity of the proposed method, a method based on fuzzy clustering algorithm is proposed to analyze the human-land coupled bearing capacity of Qiangtang meadow in northern Tibet. 


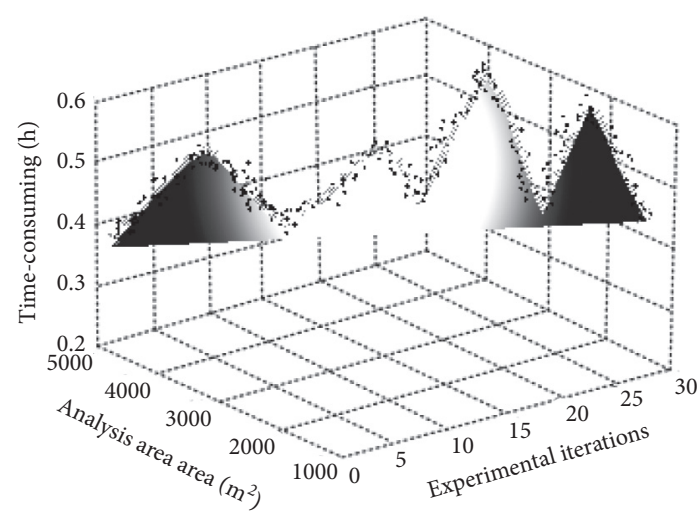

(a)

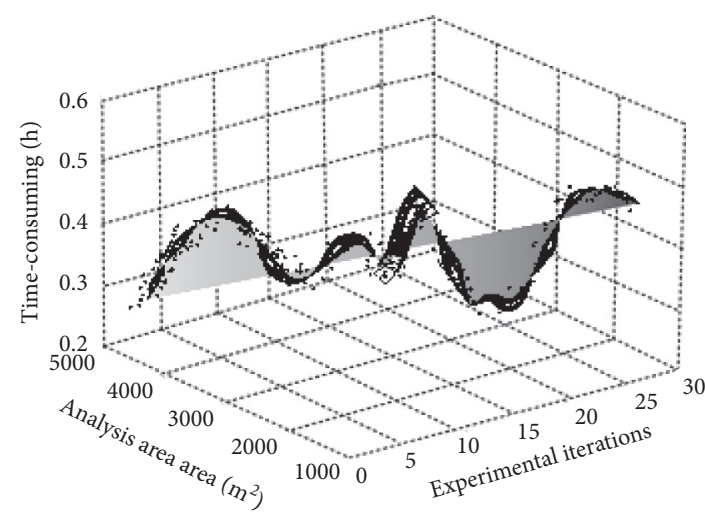

(b)

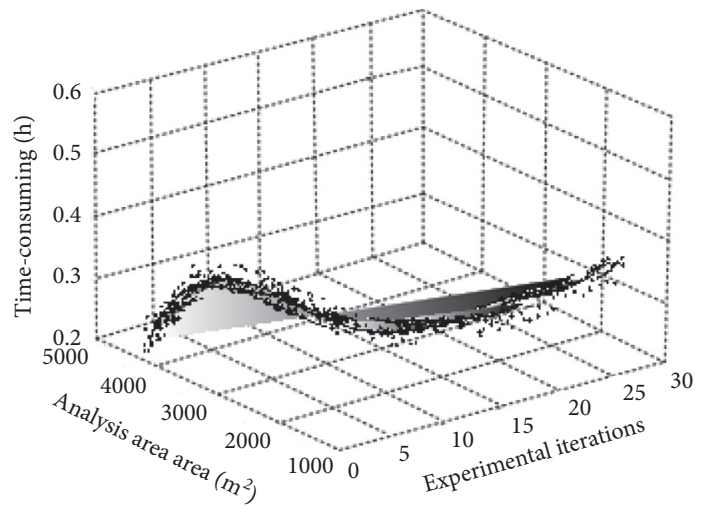

(c)

Figure 3: Comparisons of operational efficiency of different methods. (a) Operating efficiency of method 2. (b) Operating efficiency of method 4. (c) Operating efficiency of the human-land coupled bearing capacity analysis method for Qiangtang meadow in northern Tibet based on fuzzy clustering algorithm.

The experimental platform was built on MATLAB, and the simulation of Qiangtang meadow in northern Tibet was completed by using the collected data. The method was validated in the following aspects:

(1) Analysis of energy consumption

(2) Analysis efficiency

(3) Correlation between the analysis results and the actual results

(4) Rationality analysis of the selecting indexes

The experimental results show the method proposed in references [5-8] as methods $1,2,3$, and 4, respectively. The experimental results are as follows.

Analysis in Figure 2 shows that the running energy consumption of this method is generally high. The running energy consumption of method 1 continues to increase with the increase of the analysis time and is relatively flat when the running time is $7 \mathrm{~h}$. Method 2 has low initial operation energy consumption, but it has been in the state of increasing with time, which does not have reliability. In the early stage of operation, the energy consumption curve is relatively smooth, but it does not have sustainability. The average running energy consumption of the method based on fuzzy clustering is $81 \mathrm{~J}$, which is lower than the current method.
From Figure 3, it can be clearly seen that the method based on the fuzzy clustering algorithm has a high operational efficiency under different study areas and the number of experimental iterations. The main reason for this result is that after collecting the geographical information data of Tibet, the image data collected are processed by means of pretreatment, which effectively improves the efficiency of the method.

From Figure 4, it can be seen that the results of the human-land coupled bearing capacity analysis of Qiangtang meadow in northern Tibet based on the fuzzy clustering algorithm are consistently above $85 \%$, and the highest is $97 \%$. It can be clearly seen from the figure that the proposed method is very robust. The method can effectively improve the fitting degree between the analyzed results and the actual situation of the human-land coupled bearing capacity of Qiangtang meadow by using fuzzy clustering, bearing capacity evaluation, and bearing capacity.

In Figure 5, the rationality coefficient of the proposed method is relatively high. This method divides the evaluation index system of Qiangtang meadow carrying capacity into three parts: population resources subsystem, natural resources subsystem, and environment resources subsystem. According to the Delphi method and the opinions of 20 experts on the importance ranking and weight assignment of 


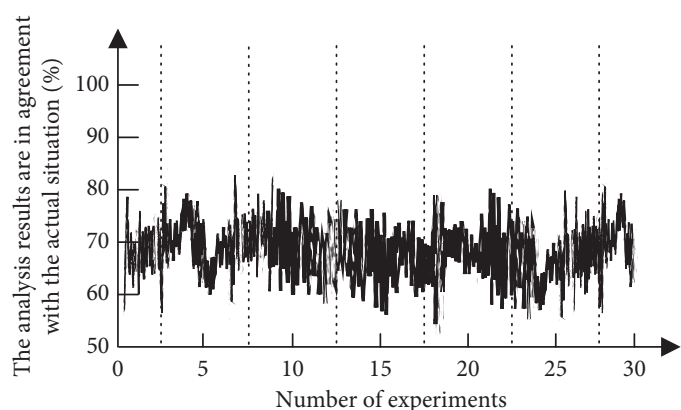

(a)

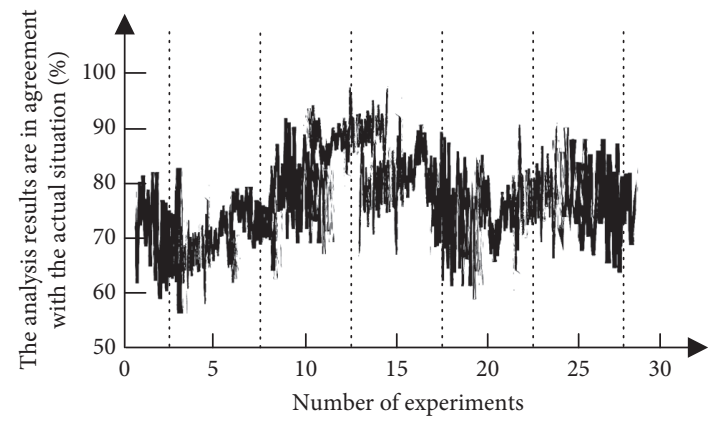

(c)

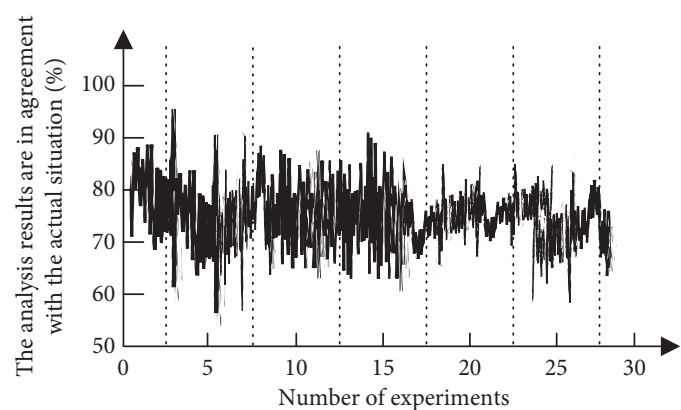

(b)

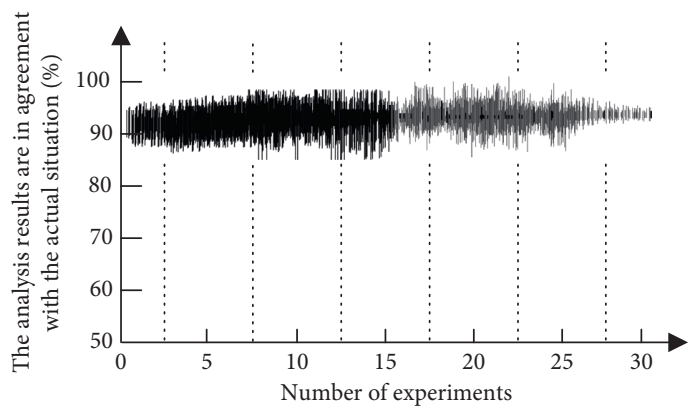

(d)

Figure 4: Comparisons between the results of different methods and actual situations. (a) Correlation between the analytical results of method 3 and the actual situation. (b) Correlation between the analytical results of method 1 and the actual situation. (c) Correlation between the analytical results of method 2 and the actual situation. (d) The results of human-land coupled bearing capacity analysis of Qiangtang meadow in northern Tibet based on fuzzy clustering algorithm are consistent with the actual situation.

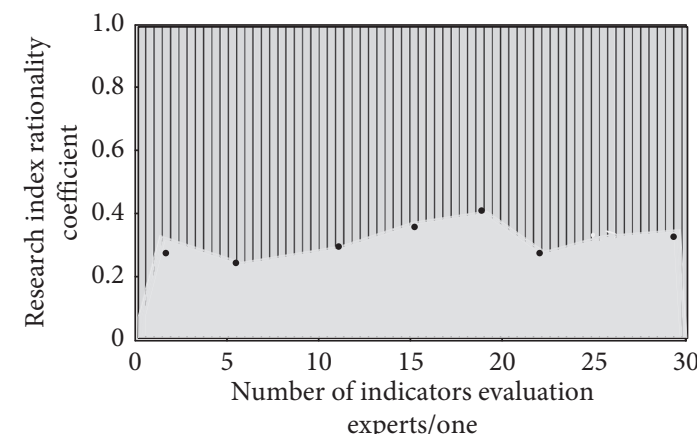

(a)

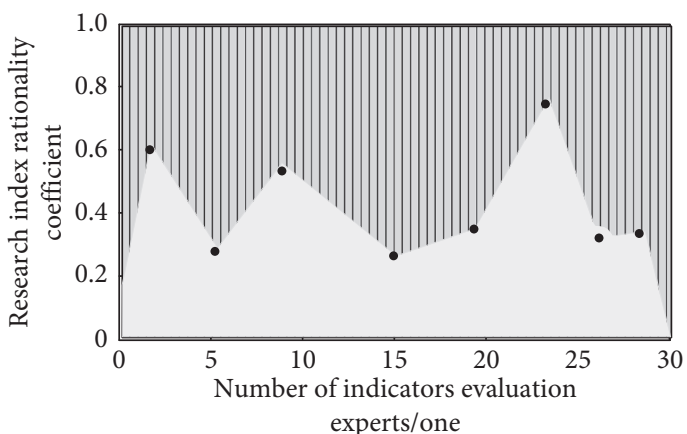

(c)

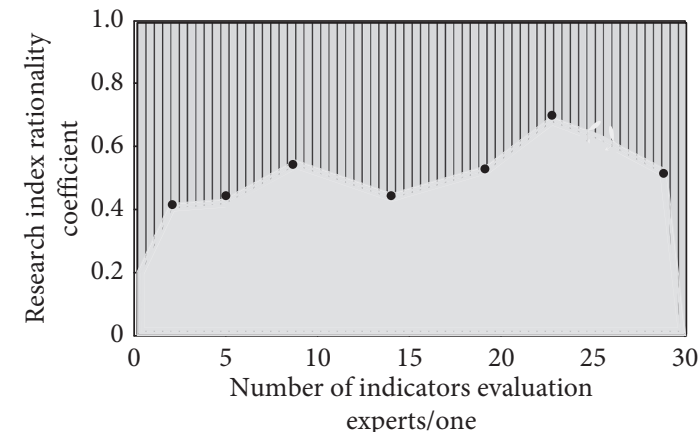

(b)

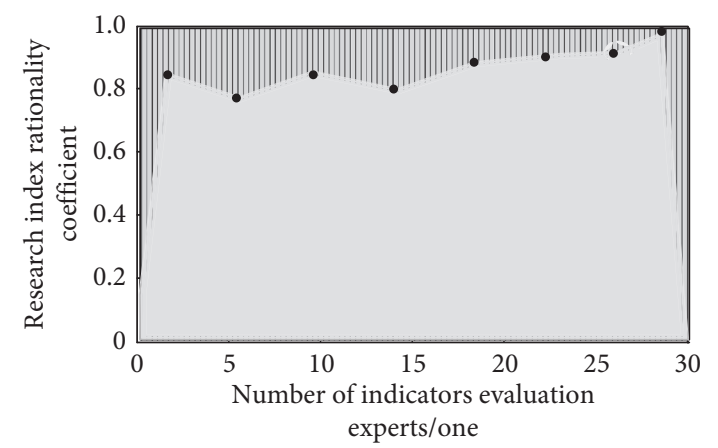

(d)

FIGURE 5: Rationality of indexes by different methods. Calculation considering the closure effect. (a) Rationality of the index in research method 4. (b) Rationality of the index in research method 2. (c) Rationality of the index in research method 3. (d) Rationality of indexes for the analytical method of man-land coupled bearing capacity of Qiangtang meadow in northern Tibet based on fuzzy clustering algorithm. 


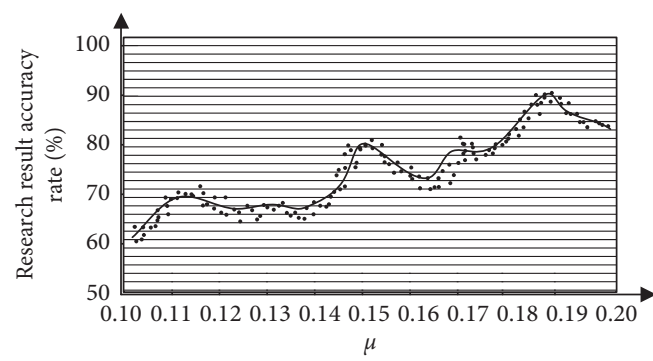

(a)

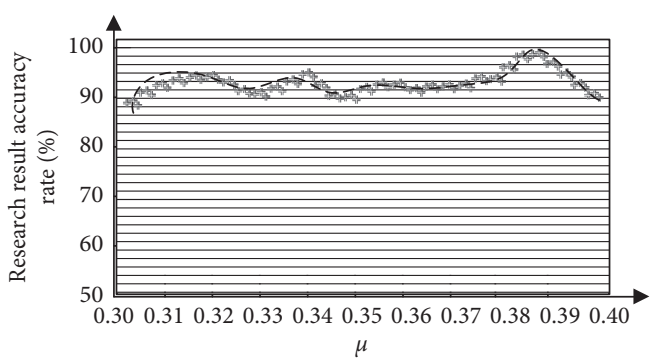

(b)

FIGURE 6: The influence of different values of $\mu$ on the accuracy of human-land coupled bearing capacity analysis of Qiangtang meadow in northern Tibet. (a) Accuracy of human-land coupled bearing capacity analysis of Qiangtang meadow in northern Tibet when $\mu$ is within the interval of $[0.1,0.2]$. (b) Accuracy of human-land coupled bearing capacity analysis of Qiangtang meadow in northern Tibet when $\mu$ is within the interval of $[0.3,0.4]$.

the index system, the weight values at all levels of the index system were determined. Thus, the rationality of the research index is improved.

\section{Discussion}

The regional cluster adjustment threshold $(\mu)$ of bearing capacity is defined as the object, and its value is divided in $[0.1,0.2]$ and $[0.3,0.4]$ in order to observe the effect of $\mu$ on the accuracy of human-land coupled bearing capacity analysis of Qiangtang meadow in northern Tibet. The results are as follows.

It can be seen from Figure 6 that the accuracy curve of the analysis of the human-land coupled bearing capacity shows an upward trend when $\mu$ is within the interval of [0.1, 0.2 ], and it is generally not ideal; however, the accuracy of the analysis of human-land coupled bearing capacity of the meadow fluctuates slightly when $\mu$ is in the interval $[0.3,0.4]$, and the overall accuracy is higher. Through this comparison, it is proved that when the value of $\mu$ is 0.39 , the accuracy of the analysis of the human-land coupled bearing capacity can reach the highest value.

\section{Conclusion}

Grazing is the most common way of grassland utilization in the alpine region of the Qinghai-Tibet Plateau, and it is an important factor affecting the species diversity and biomass of grassland vegetation community. However, Cordyceps sinensis excavation and excessive artificial yak grazing have become a hot topic in the present scenario. Aiming at the problems existing in the current research on carrying capacity in this field, a method of human-land coupled carrying capacity analysis of Qiangtang meadow in northern Tibet based on fuzzy clustering algorithm is proposed. Experiments show that the proposed method has strong practicability. The suggestions for overuse of meadow are as follows: moderate grazing treatment can increase plant diversity in alpine meadows. Compared with zero grazing, moderate grazing can reduce the competition of dominant species, facilitating the establishment of alien species, and thus promoting the increase of species diversity. Proper excavation of Cordyceps sinensis is beneficial to activities on meadow-land and growth of meadows.

Suggestions for future research are as follows: due to the fewer flux observation stations and the limitation of data and further studies can be improved in this respect, the collection of spatialized data is still relatively small, and this aspect should be improved.

\section{Data Availability}

The data used to support the findings of this study are available from the corresponding author upon request.

\section{Conflicts of Interest}

The authors declare that there are no conflicts of interest regarding the publication of this paper.

\section{Acknowledgments}

This research was supported by the Natural Science Foundation of Tibet Autonomous Region under Grant no. ZRKX2019000302, the Youth Training Program of Tibet University under Grant no. ZDQMJH18-13, and the HighLevel Talents Training Program of Tibet University under Grant no. 2017-GSP-019.

\section{References}

[1] J. T. Wang and D. Jin, "Research on cloud data distribution law based on fuzzy clustering analysis," Automation \& Instrumentation, vol. 10, pp. 11-12, 2017.

[2] Y. Q. Zhang and J. L. Tang, "Clustering sorting algorithm based on digital channelized receiver," Journal of China Academy of Electronics and Information Technology, vol. 12, no. 2, pp. 143-148, 2017.

[3] M. Y. Zhu, L. H. Feng, F. Xiao et al., "Study on the carrying capacity of water resources in anhui province on the principal component analysis," Bulletin of Science and Technology, vol. 32, no. 9, pp. 26-29, 2016.

[4] S. R. Wang and Y. Chen, "Land balance and ecological environmental bearing capacity of the coupling relationship," Science Technology and Engineering, vol. 17, no. 14, pp. 338342, 2017. 
[5] M. W. Hu and P. F. Huang, "Evaluation of the recreation environmental carrying capacity of mountain meadow: taking Wugong mountain scenic area as an example," Acta Agriculturae Universitatis Jiangxiensis (Natural Sciences Edition), vol. 38, no. 6, pp. 1196-1204, 2016.

[6] R. L. Feng, L. J. Wu, Y. P. Shen et al., "Study on stress distribution rule of meadow soil ground under embankment," China Journal of Highway and Transport, vol. 29, no. 1, pp. 29-35, 2016.

[7] F. D. Sun, Y. Qing, C. Zhu et al., "Analysis of soil enzyme activities and microbial community characteristics in degraded alpine grassland, Zoige, southwest China," Journal of Arid Land Resources and Environment, vol. 30, no. 7, pp. 119-125, 2016.

[8] Z. H. Zhao, Z. R. Xu, S. K. Cheng et al., "Attribution analysis of land use/cover change in carbon accumulation dynamics in Tibet ecosystem," Journal of Natural Resources, vol. 31, no. 5, pp. 755-766, 2016.

[9] X. Hao and Y. D. Liu, "Strength of concrete filled steel tube under fatigue load prediction model simulation analysis," Computer Simulation, vol. 34, no. 3, pp. 361-364, 2017.

[10] Y. Q. Sun, Y. Y. Li, and Y. Lu, "Optimization of K-means clustering algorithm based on MapReduce," Computer Measurement \& Control, vol. 24, no. 7, pp. 272-275, 2016.

[11] D. Q. Wang and X. X. Wang, "Large data optimization particle swarm clustering algorithm based on cloud storage," Electronic Design Engineering, vol. 25, no. 2, pp. 26-30, 2017.

[12] Q. Yang, F. Zhang, Z. Jiang et al., "Assessment of water resource carrying capacity in karst area of Southwest China," Environmental Earth Sciences, vol. 75, no. 1, pp. 1-8, 2016.

[13] H. Iijima and M. Ueno, "Spatial heterogeneity in the carrying capacity of sika deer in Japan," Journal of Mammalogy, vol. 97, no. 3, pp. 734-743, 2016.

[14] S. A. Hartmann, S. Oppel, G. Segelbacher, M. E. Juiña, and H. M. Schaefer, "Decline in territory size and fecundity as a response to carrying capacity in an endangered songbird," Oecologia, vol. 183, no. 2, pp. 597-606, 2017.

[15] F. Accatino, D. Ward, K. Wiegand, and C. De Michele, "Carrying capacity in arid rangelands during droughts: the role of temporal and spatial thresholds," Animal, vol. 11, no. 2, pp. 309-317, 2017. 